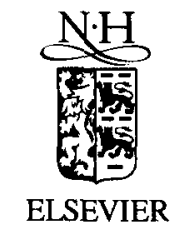

Journal of Nuclear Materials $241-243$ (1997) $483-488$

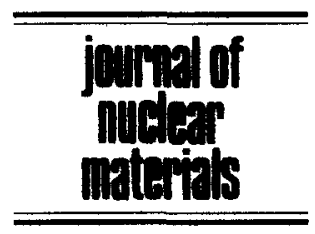

\title{
Arc jets for divertor component testing
}

\author{
John Balboni $^{\text {a, * }}$, Samuel A. Cohen ${ }^{\text {b }}$, Tobin Munsat ${ }^{b}$ \\ ${ }^{a}$ NASA Ames Research Center, Moffett Field, CA 94035, USA \\ b Princeton University, Plasma Physics Laboratory, Princeton, NJ 08543, USA
}

\begin{abstract}
This paper describes the potential for arc jet facilities to simulate the thermal and chemical environment inside a fusion-reactor power and particle exhaust section to provide the fusion materials science and plasma physics communities with data on their models and component designs.

Keywords: Divertor plasma; Divertor simulator; Plasma-wall interactions simulator; Fusion technology
\end{abstract}

\section{Introduction}

Power and gas exhaust components, typified by divertors in tokamaks, include thermal protection tiles, support and cooling structures, and vacuum pumps. These will require extensive testing to qualify them for use in MFE reactors and prototype reactors. A large high-power arc jet plasma facility may provide a representative means for extensive thermal testing of such components in a warm hydrogen environment, as expected in the divertor regions of tokamaks or end sections of linear plasma devices. This new application of arc jets could benefit the international thermonuclear experimental reactor program, ITER, of which the United States is a partner. In this paper we concentrate on tokamak divertor applications, though similar results would be found for linear fusion devices.

Pressure/energy constraints on the parameters expected in the scrape-off layer (SOL) of MFE reactors indicate that the peak power flow along the magnetic field will be $\sim 2 \mathrm{GW} / \mathrm{m}^{2}$, corresponding to $\sim 0.1 \mathrm{GW} / \mathrm{m}^{2}$ in the poloidal direction and $10 \mathrm{MW} / \mathrm{m}^{2}$ onto tilted divertor plates in tokamaks. Transients could cause 10 times greater heat loads. In the SOL, the plasma plus neutral densitytemperature product (pressure) near material surfaces may reach $10^{22} \mathrm{~m}^{-3} \mathrm{eV} \sim 10$ Torr. Attaining divertor plasma temperatures below $5 \mathrm{eV}$ is considered essential to avoid

\footnotetext{
* Corresponding author. Tel.: + 1-415 604 6064; fax: + 1-415 604 5244; e-mail: jbalboni@mail.arc.nasa.gov.
}

sputter erosion, so the envisioned divertor operational regimes have a high density of atomic hydrogen. The effects on PFC materials and structures of continuous long-term exposure to such streams of hot reactive gases have not been studied. As described below, arc jets can provide such an environment and the means to carry out divertor materials and component testing.

Arc-heated wind tunnels, or arc jets, have been employed by the aerospace research community for many years to reproduce the aerothermal environment of highspeed flight in the atmosphere of Earth and other planets. Several such facilities exist at the Ames research center operated by NASA. They are used for thermal protection materials development and certification for space vehicles [1]. Input power levels up to $80 \mathrm{MW}$ have been demonstrated in these arc jets. The giant planet facility, GPF, served as the primary source of thermal design data during development of the heat shield for the Galileo probe [2] which survived entry into the Jovian atmosphere in December 1995. The composition of the atmospheres of Jupiter and all of the outer planets is predominantly hydrogen-helium, in a ratio similar to that expected in fusion reactor exhaust streams.

\section{Comparison of ITER divertor and GPF parameters}

Table 1 lists the main characteristics of the GPF and the ITER divertor power and particle exhaust systems. A major difference between the arc jet and the ITER divertor 
Table I

Comparison between the ITER SOL and the GPF

\begin{tabular}{|c|c|c|}
\hline Characteristics & ITER requirement & Arc jet capability \\
\hline Power input & $100 \mathrm{MW}$ to SOL & $80 \mathrm{MW}$ maximum \\
\hline Enthalpy $(\mathrm{MJ} / \mathrm{kg})$ & 3 (midplane) & 10 to 400 \\
\hline Peak heat flow $\left(\mathrm{GW} / \mathrm{m}^{2}\right)$ & $2\left(\mathrm{~B}_{\mid \mathrm{I}-\mathrm{SOl}}\right)$ & $10($ axial $)$ \\
\hline Plasma flow (Mach number) & $\sim 1$ & $1-10$ \\
\hline$T_{\mathrm{e}}, T_{\mathrm{i}}$ & $1-2 \mathrm{eV}$ desired & $1-2 \mathrm{eV}$ obtained \\
\hline Power to side walls $\left(\mathrm{MW} / \mathrm{m}^{2}\right)$ & turbulence desired & 1-30 (laminar/trans.) \\
\hline Pulse length $(\min )$ & 15 & 30 maximum \\
\hline Pulses & 10,000 in life & $1000 /$ year \\
\hline Cyclic heat load $(\mathrm{Hz})$ & 0.1 (sweeping) & TBD \\
\hline Hydrogen content & $90 \mathrm{H}-10 \mathrm{He}$ & to $100 \%$ \\
\hline Chemical erosion & expected & observed \\
\hline Test area $\left(\mathrm{m}^{2}\right)$ & $>1$ & $0.1-0.5$ \\
\hline Plasma width $(\mathrm{m})$ & $\sim 0.1$ & $0.07 \rightarrow 1 \mathrm{~m}$ \\
\hline Pumping speed $\left(\mathrm{m}^{3} / \mathrm{s}\right)$ & 500 & $>500$ (non-combustible) \\
\hline Throughput $(\mathrm{kg} / \mathrm{s})$ & 0.20 & $0.1-10$ (non-combustible) \\
\hline
\end{tabular}

is the absence of a magnetic field in the former. Hence arc jets are not suitable to test plasma physics effects dominated by the magnetic field. Detached plasmas, the presently chosen divertor operational scenario, are likely to behave as unmagnetized plasmas because of the relevant ratios of scale lengths and collision frequencies. Other shortcomings are described in Section 3.

Heat loads to a large test article (ca. $1 \mathrm{~m}$ ), as will be experienced by components in the ITER divertor, can be matched in the GPF using the splash test technique (Fig. 1). Continuous surface heat loads of $30 \mathrm{MW} / \mathrm{m}^{2}$ have been demonstrated in hydrogen on a $30 \mathrm{~cm}$ diameter body at one atmosphere. Hydrogen plasma density at the surface of the test article would be of the order of $10^{24} \mathrm{~m}^{-3}$ at this pressure. If, however, vacuum exhaust of non-combustible plasma is utilized, number densities a factor of 10 to 100 lower can be attained. Numerical simulations of lower density operation is in progress. Duty cycles of $30 \mathrm{~min}$ on,
30 min off would allow multiple exposures per day resulting in a capability for an extensive testing program.

\section{GPF arc jet facility}

The GPF is a direct-current arc-discharge device designed to heat flowing hydrogen-helium mixtures up to $10000 \mathrm{~K}$ at $5 \mathrm{~atm}$ reservoir pressure for a period of minutes. The flowing plasma, up to $0.2 \mathrm{~kg} / \mathrm{s}$, expands into a chamber where a test article is exposed to the flow. Test articles of $30 \mathrm{~cm}$ diameter have been tested in hydrogen helium mixtures at steady state surface heat loading of 30 $\mathrm{MW} / \mathrm{m}^{2}$ (splash test) and on a $4 \mathrm{~cm}$ diameter body (fully immersed) up to $260 \mathrm{MW} / \mathrm{m}^{2}$. Such heat loads and compositional mixes are expected in the ITER divertor. Plasmas of various mixtures of hydrogen, helium, and other

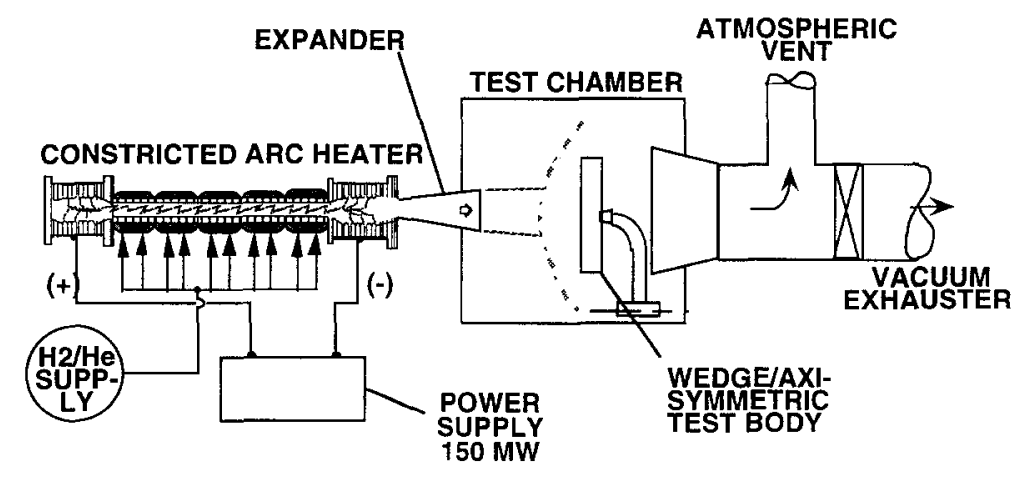

Fig. 1. Diagram of the giant planet facility arc jet. 


\section{GIANT PLANET FACILITY}

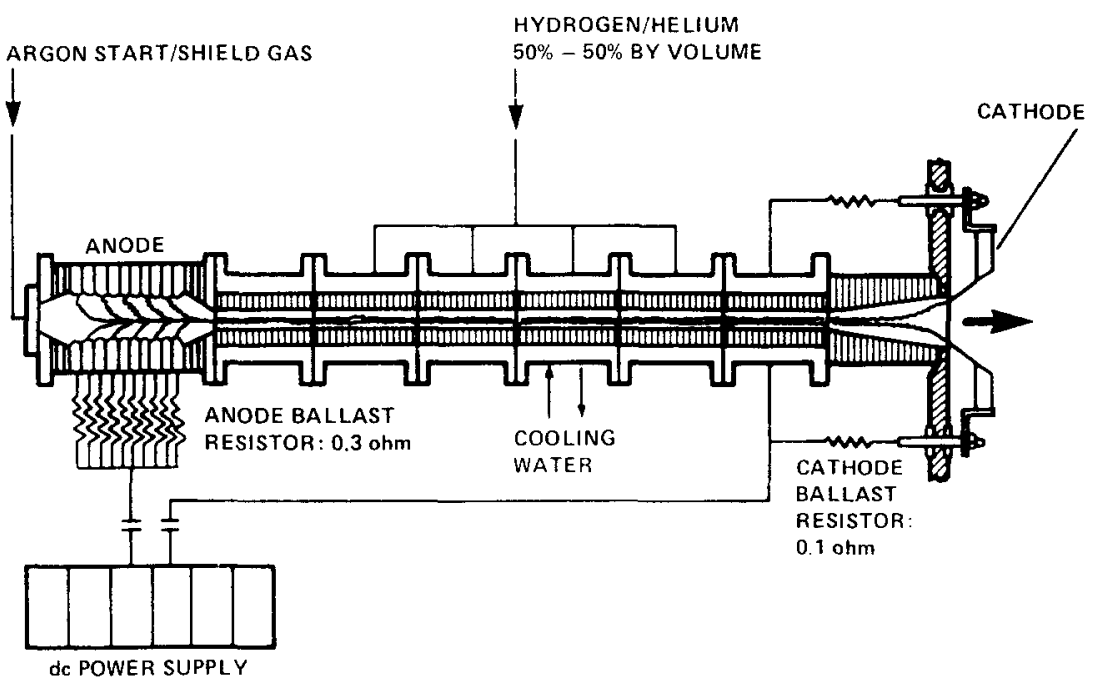

Fig. 2. Constricted-arc heater details.

gases can be heated in the GPF. Capability exists for vacuum pumping of the test chamber when non-combustible gas mixtures are used. Installation (and testing) of ITER pumps, in place of the usual arc jet pumping system, would allow continuous use of the arc jets at lower chamber pressures, similar to certain operational modes predicted for ITER.

The GPF was placed into storage in 1988 pending future hydrogen plasma testing needs. Reassembly would be necessary to conduct thermal tests on ITER divertor components under steady-state or transient heat loads in hydrogen or other plasmas. Present considerations of proposed future NASA missions to the outer planets also include consideration for a hydrogen arc jet such as GPF. Reactivating the GPF would require some refurbishment of portions of the arc jet support subsystems that are shared with five other operating arc jets at Ames. Plasma characterization research in the form of experimental and computational tools is being conducted which could help to assess the plasma environment in the proposed test facility, and aid in analyzing results.

\subsection{Jovian entry}

Atmospheric entry missions to the outer planets have received renewed interest following the success of the Galileo probe in December 1995. The GPF was originally constructed [3] to simulate the thermal heat load and chemical environment experienced by the Galileo probe as it entered the atmosphere of Jupiter at a relative velocity of $48 \mathrm{~km} / \mathrm{s}$. During the deceleration of the $340 \mathrm{~kg}$ probe most of its kinetic energy is dissipated into the atmosphere. A small portion of the kinetic energy, about $0.5 \%$, or 1.9
GJ, was absorbed by the probe during the $20 \mathrm{~s}$ heating interval, necessitating heat shielding for the internal instrumentation [4]. A $1.3 \mathrm{~m}$ diameter carbon phenolic heat shield comprised $45 \%$ of the total mass of the probe. Ablation of the carbonaceous heat shield material protected the probe's internal components. Under the applied convective heat load at the nose, $155 \mathrm{MW} / \mathrm{m}^{2}$ maximum, the heat shield thickness receded $9 \mathrm{~cm}$. A majority of the energy radiated from the hot shock layer in front of the nose, predicted to peak at $510 \mathrm{MW} / \mathrm{m}^{2}$, was absorbed by the layer of ablation gases and thus blocked from being absorbed by the nose cone itself. During the probe design phase in the mid 1970's, data was required which described the thermochemical ablation of carbon phenolic in a hydrogen-helium atmosphere in order to optimize the heat shield thickness and thus reduce the heat shield mass. This was the basis for building the giant planet facility.

\subsubsection{GPF experience}

The giant planet facility arc jet was constructed in 1975 to address the lack of ablation recession data in a hydrogen-helium atmosphere during the design of the Jovian entry probe [2]. The diagram in Fig. 2 depicts GPF with nine water-cooled copper anode rings and four carbon cathode rods. Series ballast resistors help stabilize and equalize current to each electrode. The test gas is heated by proximity to a direct-current arc discharge confined within a cylindrical tube, called the constrictor column. The $6 \mathrm{~cm}$ diameter constrictor column is segmented into 432 disks, each $1 \mathrm{~cm}$ thick. Each disk is electrically isolated and contains gas injection and water cooling passages. Anode and cathode materials, typically copper, carbon, or thoriated tungsten, are bathed in argon or nitrogen 
gas which increases their operating life to tens of hours. Cooling water removes waste heat and maintains the integrity of the arc heater components. Open at one end, the plasma flows from the constrictor through an expander into a test chamber where a material sample is immersed in the stream. Power density in the plasma stream can reach $10 \mathrm{GW} / \mathrm{m}^{2}$ at maximum input power of $80 \mathrm{MW}$ after approximately $65 \%$ of the energy is lost to the walls of the constrictor. The expansion ratio of the conical GPF nozzle was 1.36 , producing a plasma stream diameter of 7 $\mathrm{cm}$. This expansion ratio produces a balanced plasma jet pressure of one atmosphere in the test chamber, compatible with the requirement that the hydrogen plasma be exhausted to the atmosphere. Maximum duration of a run is $30 \mathrm{~min}$, controlled by the temperature rise of components in the dc power supply, and several runs per day are possible. For non-combustible gases a vacuum pumping system maintains sub-atmospheric pressure in the test chamber. Hydrogen plasma exhausts to atmosphere, although non-combustible plasmas can be pumped by the vacuum exhaust system which can maintain the test chamber pressure at about 10 to 50 Torr. Electrical power, cooling water, instrumentation, and gas subsystems all are shared by the five operating arc heated facilities in the complex.

Three potential shortcomings of ITER testing in GPF are mentioned here. First, there is a possibility for contaminating the test article by sputtered material from the electrodes in the arc heater (copper, tungsten, and carbon are typical electrode materials). However, concentration levels of copper and tungsten in the core of the plasma have been determined to be small, of the order of parts per million. There is evidence that a majority of these contaminants remain in particulate form at the edges of the plasma stream. Second, atmospheric pressure in the test chamber is required to prevent explosive gas mixtures from entering the vacuum pumping system. Sub-atmospheric pressures could be achieved with non-explosive plasmas as the test gas, or with an auxiliary vacuum pump that, though, not defined at present, might be a prototype ITER pump. Finally, beryllium and its oxides cannot be released within the test chamber or the vacuum system.

\subsubsection{Atmospheric exhaust testing}

Nearly all GPF tests were conducted with the test chamber at 1.05 bar to allow for atmospheric exhaust of the hydrogen. The simulation requirement from the Galileo probe trajectory is overlaid with the GPF operating envelope (Fig. 3). Two axial locations for test articles were available in GPF, $7.6 \mathrm{~cm}$ and $76 \mathrm{~cm}$ distance from the nozzle exit plane, to simulate the environment at the nose and the afterbody regions of the Galileo probe, respectively. Test articles in both locations were of the stagnation flow configuration. In the former, a $4 \mathrm{~cm}$ diameter test body was fully immersed in the plasma stream of diameter

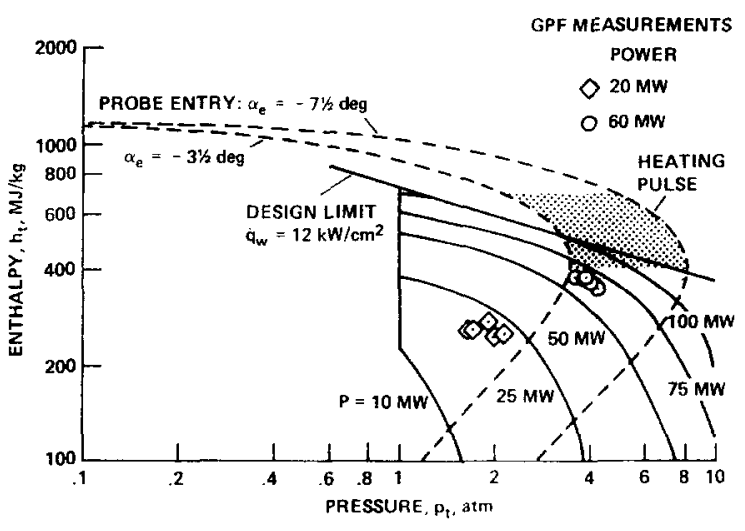

Fig. 3. Operating envelope for the GPF arc jet with a $50 \%$ hydrogen $-50 \%$ helium gas mixture. Overlaid is the Galileo probe entry trajectory corridor [2]

$7 \mathrm{~cm}$. Recession rate and shape change data of carbon phenolic were obtained at the highest heat flux levels, up to $260 \mathrm{MW} / \mathrm{m}^{2}$. At the $76 \mathrm{~cm}$ diameter station, at which point the plasma stream had further expanded from $7 \mathrm{~cm}$ diameter to approximately $10 \mathrm{~cm}$, a $30 \mathrm{~cm}$ diameter test body was inserted into the plasma stream. The technique of testing a body larger than the flow stream is called a splash test. Peak surface heat flux of $30 \mathrm{MW} / \mathrm{m}^{2}$ was attained in the splash test configuration at surface pressures of $1 / 2$ to 1 atmosphere. Variations of about $50 \%$ in heat flux and pressure across the front surface of the body were considered acceptable. The constraint that the hydrogen must exhaust from the test chamber constrains the degree of expansion of the plasma, and therefore the size of the plasma stream. The splash test is the technique which accommodates the largest test article in hydrogen, therefore is, at present, the recommended test technique for large ITER divertor components in hydrogen.

\subsubsection{Vacuum exhaust testing}

The capability for pumping the plasma exists for noncombustible mixtures. At $0.2 \mathrm{~kg} / \mathrm{s}$ flow rate the test chamber pressure can be maintained at 10 to 50 Torr using the five-stage steam ejector pumping system. This system is routinely used to simulate high altitude flow environments, and is shared between all operating arc jet facilities in the Ames complex. Flow rates as high as $10 \mathrm{~kg} / \mathrm{s}$ are possible at approximately $1 / 2$ bar. It is possible to generate larger plasma streams with the vacuum exhauster by using an expander nozzle. Expansion ratios up to 300 (by area) are possible. Panels up to $60 \times 60 \mathrm{~cm}$ and bodies up to $40 \mathrm{~cm}$ diameter are routinely testing in air/nitrogen/argon plasmas. Further analysis, possibly followed by pump development, is needed to determine which test technique, atmospheric or vacuum exhaust, is best suited for ITER testing in arc jet facilities. 


\subsection{Plasma characterization in GPF}

Characterization of the plasma flow in GPF will be a valuable tool in assessing the effect of the plasma environment on the surface of the test article. Due to various physical phenomena inherent to the process of producing plasma in arc jets, the heat content of the hypersonic gas is not distributed according to the laws of thermochemical and thermodynamic equilibrium. Some of the energy is disproportionately distributed among the vibrational, rotational and electronic states. This problem is further complicated by the existence of a thermochemical nonequilibrium in the flow field. In order to properly assess the heat bearing capabilities of a test article we must characterize the flow field. In doing so, we also need to quantify the significance of this nonequilibrium phenomenon on the heat transfer processes in the plasma. Such phenomena are inherently of interest to ITER because of the relation to detached (thermally collapsed) [5] plasma operation.

Currently, an effort at NASA Ames is underway to develop experimental diagnostics and computational tools that can characterize the flow field of an arc jet [6]. The experimental efforts are directed to three areas: (1) accurate estimation of operational parameters, such as flow enthalpy, pressure distribution, transient effects and repeatability of the test conditions, (2) characterization of the flow field parameters, such as the radiative component of heat flux, vibrational, rotational and electronic temperatures of various gas species, flow velocity, and species concentration, and (3) measurements of various chemical rates effecting the heat transfer such as, atomic recombination rates at the model surface and vibrational relaxation rates. The issue of spatial non-uniformity in the flow field is also being addressed.

Spectroscopic emission measurements are made to determine the radiative component of the heat flux, to measure vibrational, rotational and electronic temperatures of various radiative species, and to determine the species concentration of various atomic species. Experimental characterization of the hydrogen plasma would be directed to three regions of the arc jet: the plenum, the free stream, and the shock layer in front of the test body. Spectrally-resolved emission measurements from the plenum region would be used to assess the state of the plasma before it enters the nozzle. In the free stream region, laser-based spectroscopic instrumentation would be employed to determine flow velocity, translational temperature, and dissociation level. The approach used would be two-photon excitation of atomic hydrogen using a frequency doubled $\mathrm{Nd}$ :YAG-pumped dye laser system. The $\mathrm{H}$ atom concentration (dissociation level) depends on the total fluorescence signal level and the transition spectral line width depends on translational temperature. By orienting the laser beam at an angle less than $90^{\circ}$ to the axis, the absorption frequency of the transition becomes sensitive to the axial flow velocity through the Doppler effect. A similar strategy has been used to obtain the same flow property measurements another arc heated facility using two-photon, laser-induced fluorescence of atomic oxygen and nitrogen. Finally, spectrally resolved emission measurements from the shock layer over the model surface could also be used to help characterize the flow within the layer. Electronic temperatures could be determined from Boltzmann plots of the $\mathrm{H}$ atom emission, and electron population measurements could be attempted from Stark-broadened line width measurements of appropriate $\mathrm{H}$ lines.

Data from the experimental measurements would be used in conjunction with computational flow and chemistry models to optimize the facility operation for the intended application. Computational simulations can be divided into two groups: (1) Simulation and performance analysis of energy deposition process by the electric arc, and (2) simulation of the flow field i.e., nozzle flow and the flow around the test article. ARCFLO 4 code is used to study the arc heater performance. ARCFLO II can generate flow field parameters, such as temperature, velocity, and enthalpy profile in the plenum chamber (situated at the exit of the arc heater) based on the operational parameters of the arc jet, such as arc current, voltage, chamber pressure and gas flow etc. The code uses an axisymmetric Joule heating model for the arc heating process and a 1D model for the radiative transport model within the arc constrictor tube. The radiative transport model uses a simplified twoband emission/absorption model to simplify the computation and reduce the CPU time. The ARCFLO code was utilized extensively during the Galileo heat shield development program using GPF operational data to compute the flow field parameters in the arc constrictor region ${ }^{6}$.

The existing blunt body (NEQ2DBLNTB) and nozzle (NEQ2DNZZL) codes are used to simulate the arc jet flow field. These codes are basically research codes that solve 2D/axisymmetric Navier-Stokes equations for air flows with thermochemical nonequilibrium processes using a fully implicit Gauss-Seidel line relaxation method. In the present formulation, the nonequilibrium gas model for air consists of eleven or twelve (with Argon) chemical species, $\left(\mathrm{N}_{2}, \mathrm{O}_{2}, \mathrm{NO}, \mathrm{N}, \mathrm{O}, \mathrm{N}_{2}^{+}, \mathrm{O}_{2}^{+}, \mathrm{NO}^{+}, \mathrm{N}^{+}, \mathrm{O}^{+}, \mathrm{e}^{-}, \mathrm{Ar}\right)$, and the thermal state of the gas is described by multiple temperatures: translational, rotational and vibrational (vibrational-electronic). Argon is included in the thermochemistry model of air for are jet flow environments. Nozzle and blunt body codes: NEQ2DBLNTB codes include the boundary conditions to account for surface catalysis. For the hydrogen plasma the code can be modified to include various hydrogen derivatives, such as $\mathrm{Ar}, \mathrm{H}, \mathrm{H}_{2}$, $\mathrm{H}^{+}$etc.

\section{Summary}

Arc jet facilities are shown to be capable of providing a plasma environment similar to that inside the divertor 
region of fusion devices. Existing arc jet technology can be applied to perform thermal tests for the ITER program. Flowing hydrogen plasma at approximately $1 \mathrm{eV}$ can be generated for $30 \mathrm{~min}$, several times per day. High power arc jets have been developed by NASA for atmospheric entry simulation up to the $80 \mathrm{MW}$ class. One such facility, the giant planet facility, was used to simulate the entry environment in the hydrogen-helium atmosphere of Jupiter during the design/development of the heat shield for the Galileo entry probe. The facility has been deactivated and placed in storage. Reactivation of the GPF could provide steady state and/or transient thermal life testing on full scale ITER divertor components. The operating envelope of the GPF overlaps with the expected environment of the exposed sections of the divertor. Experience in arc jet operation and plasma characterization at NASA Ames can be applied to the proposed reactivation effort. Laser diagnostics coupled with plasma computational codes are available to design and analyze data. With increasing interest in future outer planet space missions, cooperative programs between NASA, DOE and the ITER program may be possible.

\section{References}

[1] A. Balter-Peterson et al., Arc Jet Testing in NASA Ames Research Center Thermophysics Facilities, AIAA-92-5041. AIAA 4th Int. Aerospace Planes Conf. December (1992).

[2] W. Winovich and W. Carlson, The Giant Planet Facility, 25th Instrument Soc. of America Symp. (Anaheim, CA, 1979).

[3] J. Lundell, Research and Technology Development for the Galileo Entry Probe Heat Shield, November 30, 1995, unpublished paper.

[4] S.P. Sharma et al., Arc jet Flow Characterization, AIAA-960612, Reno, NV, January 1996.

[5] G.S. Chiu and S.A. Cohen, Phys. Rev. Lett. 76 (1996) 1248, and references therein.

[6] M. Green and W. Davy, Numerical Simulation of experiments in the Giant Planet Facility, AIAA-79-1 102, AIAA 14th Thermophysics Conf., Orlando, FL, June 1979. 